SSN Impreso: 1794-9920

ISSN Electrónico: 2500-9338

Volumen $17-\mathrm{N}^{\circ} 2$

Año 2017

Págs. 110-119

\title{
INFLUENCIA DE LAS TIC EN LA COMPETITIVIDAD ORGANIZACIONAL
}

\author{
Marco Antonio Sarmiento Martínez ** \\ Enlace ORCID: http://orcid.org/0000-0003-2369-6138 \\ Akever Karina Santafé Rojas *** \\ Enlace ORCID: http://orcid.org/0000-0002-4535-3470 \\ Laura Teresa Tuta Ramírez **** \\ Enlace ORCID: http://orcid.org/0000-0003-0048-874X
}

Fecha de Recepción: 16 enero 2017

Fecha de Aprobación: 10 abril 2017

\section{Resumen:}

La investigación tuvo por finalidad diagnosticar la influencia de las TIC en la competitividad organizacional, para lo que se tomaron como referente 14 Instituciones de educación pública de las comunas 7 y 8 del municipio de San José de Cúcuta, buscando conocer cómo el componente tecnológico influye en el fortalecimiento del servicio educativo y a su vez en el desarrollo de habilidades cognitivas en los educandos para ser competentes, con el fin de que las instituciones del sector estudiado conciban estas herramientas como fundamento para una educación de calidad, estimulando la participación activa del estudiante en la búsqueda de un autoaprendizaje deseable y significativo $p$

ara que logre insertarse con éxito en la educación superior. Las bases teóricas que dieron sustento a los objetivos del estudio fueron la dimensión escuela del Instituto para el Desarrollo y la Innovación Educativa de la OEI (2009), la competencia instrumental por Escamilla (2008) y los factores de competitividad empresarial por Martínez, Santero, Sánchez \& Marcos (2009). El estudio se desarrolló en el marco descriptivo con enfoque cuantitativo de corte transversal, método (M.A.S) donde se empleó la técnica encuesta para tres muestras compuestas por 14 rectores, 103 docentes y 303 estudiantes de las comunas en mención. Se concluyó que siendo el uso de las TIC influyente en la competitividad organizacional, en las instituciones analizadas se evidencia como factor positivo.

Palabras Claves: TIC, competitividad, educación, habilidades cognitivas, comunas

\footnotetext{
** Administrador Comercial y de Sistemas. Universidad de Pamplona, Esp Tec. Diseño y Desarrollo de Investigaciones de Mercado. Servicio Nacional de Aprendizaje (SENA), correo: academicomarko@gmail.com
} 


\begin{abstract}
*** Postdoctora en Gerencia Pública y Gobierno. Postdoctora en Gerencia de las Organizaciones. Doctora en Ciencias Gerenciales. Magíster en Gerencia de Empresas. Especialista en Gestión Educativa. Administradora de Empresas. Licenciada en Comercio. Docente e Investigadora de la Universidad de Pamplona Colombia. Acreditada por COLCIENCIAS-Colombia como Investigadora Junior. e-mail: karisan19@hotmail.com

**** Postdoctora en Gerencia Pública y Gobierno. Postdoctora en Gerencia de las Organizaciones. Doctora en Ciencias Gerenciales. Magíster en Gerencia de Empresas. Especialista en Gestión Educativa. Administradora de Empresas. Licenciada en Comercio. Docente e Investigadora de la Universidad de Pamplona Colombia. Acreditada por COLCIENCIAS-Colombia como Investigadora Junior. e-mail: laurat@unipamplona.edu.co
\end{abstract}

\title{
INFLUENCE OF ICT IN ORGANIZATIONAL COMPETITIVENESS
}

\begin{abstract}
The purpose of the research was to diagnose the influence of ICTs on organizational competitiveness, for which they were taken as a reference 14 Public education institutions in communes 7 and 8 of the municipality of San José de Cúcuta, seeking to know how the technological component influences The strengthening of the educational service and, in turn, the development of cognitive abilities in the students to be competent, in order that the institutions of the studied sector conceive of these tools as a foundation for a quality education, stimulating the active participation of the student in the search for a desirable and meaningful self-learning so that it succeeds in inserting itself successfully in higher education. The theoretical bases that gave support to the objectives of the study were the school dimension by the Institute for Development and Educational Innovation of the OEI (2009), the instrumental competence by Escamilla (2008), and the factors of business competitiveness by Martínez, Santero, Sánchez \& Marcos (2009). The study was developed in the descriptive framework with a quantitative cross - sectional approach, method (M.A.S) where the technique was used for three samples composed of 14 rectors, 103 teachers and 303 students from the communes mentioned. It was concluded that because the use of ICTs was influential in organizational competitiveness, in the analyzed institutions it was possible to be seen as a positive factor.
\end{abstract}

Keywords: ICT, competitiveness, education, cognitive skills, communes.

\section{INFLUÊNCIA DAS TIC NA COMPETITIVIDADE ORGANIZACIONAL}

\begin{abstract}
Resumo
A pesquisa teve como objetivo diagnosticar a influência das TIC na competitividade organizacional, que foram tomados como referência 14 instituições de comunas de educação pública 7 e 8 do município de San José de Cúcuta, buscando saber como tecnológico influências componentes o reforço do serviço educativo e vire no desenvolvimento de habilidades cognitivas em estudantes se consideram competentes, de modo a que as instituições do sector estudados conceber essas ferramentas como base para uma educação de qualidade, incentivando a participação ativa do aluno no busca de uma auto-aprendizagem desejável e significativa para alcançar inserido com sucesso no ensino superior. As bases teóricas que apoiaram os objectivos do estudo foram a escola pelo Instituto de Desenvolvimento e Inovação Educacional IEO (2009) dimensão, a concorrência instrumental para Escamilla (2008), e factores de competitividade empresarial por Martinez, santero, Sanchez \& Marcos (2009). 0 estudo foi conduzido na abordagem quantitativa transversal quadro descritivo, método (M.A.S), onde foi utilizada a técnica de levantamento para trés amosteras compostas de 14 reitores, 103 professores e 303 alunos dos municípios em causa. Concluiuse que, enquanto a utilização das TIC influente na competitividade organizacional, instituições evidente analisada como um fator positivo.
\end{abstract}


Palavras-chave TIC, de competitividade, educação, habilidades cognitivas, comunas. 


\section{INTRODUCCIÓN:}

La presente investigación buscó ampliar el panorama de cómo impacta el uso de las TIC en la competitividad organizacional, centrando la mirada en instituciones de educación media del sector público, denominadas en adelante (IE). Estas herramientas han cambiado profundamente el proceso de enseñanza/aprendizaje del modelo educativo tradicional, a uno donde la tecnología juega un papel fundamental en la formación de los jóvenes del mañana que continuamente se ven inmersos en un mundo donde las TIC se hacen cada vez más presentes, por ende se requiere que los estudiantes sean competentes a la hora de afrontar los retos que exige la educación superior y el sector productivo.

Por el contrario, la limitación a su acceso en un mundo globalizado, inmerso en una sociedad de la información y del conocimiento, podría ampliar brechas entre los propósitos de los impactos tecnológicos y la calidad de la educación, por tanto, es imperante conocer cómo estas herramientas se vinculan a este tipo de organizaciones, dada su relevancia social, cultural y económica, es decir, el análisis desde el sistema educativo a través de sus actores, como también determinar si esta temática ha trascendido hacia los proyectos educativos institucionales PEI. Asimismo, analizar las posturas de los centros educativos frente al uso en las TIC en las prácticas pedagógicas con miras al mejoramiento del manejo de la información, la investigación y los aportes a la innovación, que permitan elevar el nivel de competitividad institucional y la calidad de la formación de competencias esenciales que faciliten la adaptación al mundo de la educación superior o en su defecto al laboral, de sus estudiantes.

Unas de las principales causas de esta problemática es la globalización, según Gurrero (2004) en materia educativa, la globalización cambia el concepto de educación y el rol del docente (...) la tecnología de la información modifica el acceso al conocimiento. (p. 348). Esto conduce a la sociedad del conocimiento, que según la Organización de los Estados Americanos (OEA, 2014) se refiere al tipo de sociedad que se necesita para competir y tener éxito frente a los cambios económicos y políticos del mundo moderno. Asimismo, se refiere a la sociedad que está bien educada, y que se basa en el conocimiento de sus ciudadanos para impulsar la innovación, el espíritu empresarial y el dinamismo de su economía.

\section{MARCO TEÓRICO:}

En este aparte, se exponen algunos referentes teóricos que dan soporte a la investigación donde se referencia las dimensiones y la disponibilidad de las TIC, la organización de la escuela para el uso de las TIC, la formación de los educadores, y la presencia de las TIC en las prácticas pedagógicas, elementos que conforman algunos objetivos del estudio.

\subsection{Dimensiones}

Las dimensiones son grupos de características referentes a los aspectos de una institución o de un sistema sobre los cuales se emite un dictamen de valor y que, en su conjunto, expresan la totalidad del objeto de investigación. (Instituto para el Desarrollo y la Innovación Educativa (IDIE) de la Organización de Estados Iberoamericanos para la Educación, la Ciencia y la Cultura (OEI), 2009, p. 27). Para el caso del estudio se asumió el uso desde los principales actores, datos de cuantificación, dimensión pedagógica.

\subsubsection{Acepción de las TIC}

Los sistemas de comunicación masivos históricos, sumados al alto impacto del internet han dado mayor significación al uso, al acceso a la información y el conocimiento en todos los ámbitos de la sociedad en el mundo. Las tecnologías de información y comunicación TIC, permiten hacer menos complejos los procesos, aumentar la eficiencia en las tareas e incrementar la calidad de vida de los individuos. Asimismo, se afirma que "Las Tecnologías de la Información y la Comunicación, también conocidas como TIC, son el conjunto de tecnologías desarrolladas para gestionar información y enviarla de un lugar a otro. Abarcan un abanico de soluciones muy amplio. Incluyen las tecnologías para almacenar información y recuperarla después, enviar y recibir información de un sitio a otro, o procesar información para poder calcular resultados y elaborar informes" (ServiciosTIC, 2006)

\subsubsection{Disponibilidad de las TIC}

El uso de la tecnología se concreta en la cantidad y calidad de infraestructura de apoyo (equipos audiovisuales, computadores, programas, software, salas de cómputo) con que cuentan las organizaciones para el desarrollo de sus actividades. Para el caso de la investigación se analiza en la práctica pedagógica y administrativa. Entre ellos, se pueden destacar variables relacionadas con cantidad de computadoras con y sin acceso a internet hay en las instituciones, en qué espacios se encuentran, cuántas computadoras hay por alumno y por turno, cómo evalúan los profesores la cantidad de computadoras que tiene la escuela; si es buena la velocidad de conexión con relación a las necesidades de uso de la escuela; las computadoras portátiles; si son para uso administrativo o pedagógico; cuántas con desperfectos u obsoletas existen; con qué periocidad son reparadas; si infiere en el trabajo del 
profesor; otros equipamientos tecnológicos además de las computadoras. (IDIE) de la (OEI), 2009, p. 44)

\subsubsection{Uso de las TIC en la Organización}

El efecto de la enseñanza-aprendizaje se evidencia con el actuar de las instituciones educativas. No obstante, la autonomía de funcionamiento interno, pese a que actúan bajo orientaciones y directivas de funcionamiento comunes, permite encontrar diferencias en la concepción y uso de las tecnologías, aplicadas o no, al proceso de formación. Por lo que es conveniente analizar varios casos para hacerse a una idea más cerca a la realidad de estas organizaciones.

Por otra parte, cuando se diseña un programa de TIC es necesario concebir los actores y circunstancias que encierra su ejecución para la efectividad en la práctica docente. Su concreción depende de un vector fundamental: las diferentes formas como las escuelas se organizan para colocar tales programas a disposición de sus educadores y estudiantes. Esto se traduce en la coordinación de los tiempos, los espacios y las relaciones que allí se dan. (IDIE) de la (OEI), 2009, p. 72)

\subsubsection{Formación en el uso de las TIC}

Otra variable que apoya o complejiza el uso delas TIC en el aula, en la práctica pedagógica está relacionada con la formación docente para el uso de las TIC, hay que partir del análisis de estas implicaciones y qué tan preparados para el abordaje de las tecnologías de información y comunicación se encuentra el profesor. Este desarrollo avanza a pasos agigantados pero no es homogéneo el acceso a su alcance y formación. Desde este contexto, cabe profundizar en la necesidad de actualizar currículos, establecer necesidades de formación y capacitación de profesores de tal forma que impacten positivamente las necesidades de los estudiantes.

Dichas necesidades van desde la capacitación para el manejo básico de máquinas y software y el desarrollo de las llamadas competencias digitales, hasta la formación en profundidad sobre la naturaleza social e histórica de la revolución de la microinformática y sus impactos sociales y acerca de las modificaciones posibles en los procesos de enseñanza y aprendizaje en contextos sociales y educativos ricos en tecnología e información. (IDIE) de la OEl, 2009, p. 90)

\subsubsection{Presencia de las TIC en las prácticas pedagógicas}

Vale resaltar la trascendencia que la educación tiene para la construcción de sociedad, futuro y humanidad. Sin embargo, la tarea de educar es también, una de las funciones más complejas y de mayor responsabilidad y compromiso que involucra la escuela, la comunidad, la familia e impacta el desarrollo social, cultural y económico de los países. Las prácticas pedagógicas dicen qué y cómo se enseña y se aprende, las relaciones establecidas entre quien enseña y quien aprende, la visión del mundo y de la educación que sustenta tales relaciones.

Desde este marco, de tal complejidad, incorporar las TIC en las prácticas pedagógicas no es suficiente, sino que es preciso entender y actuar sobre la realidad de cada contexto institucional, a partir de sus capacidades, que reflejan el nivel de competitividad que se han propuesto alcanzar. Investigar las prácticas pedagógicas docentes exige comprender la existencia y la naturaleza de posibles concepciones pedagógicas y las "culturas tecnológicas" que las sustenten. Esto posibilitará inferir las prácticas más o menos innovadoras y consistentes, aún más cuando se haga el cruzamiento con otros descriptores. (IDIE) de la (OEI), 2009, p.106)

\subsection{Competencia instrumental}

Es el conjunto de habilidades y destreza relacionadas con la búsqueda, selección, comprensión, análisis, síntesis, valoración, procesamiento y comunicación de información en diferentes lenguajes (verbal, numérico, icono, visual, gráfico y sonoro) que integra conocimientos, procesamientos y actitudes que van, desde la disposición abierta y esforzada a buscar y contrastar información hasta su transmisión en distintos soportes (impreso, audiovisual, digital o multivariable).(Escamilla, 2008, pp. 79,197)

En este sentido, las organizaciones que han apostado al alcance de indicadores de competitividad producto de la incursión en el uso y apropiación de las TIC han considerado dentro de su infraestructura el uso de sistemas informáticos como hardware, redes, software, impresoras, estableciendo la conveniencia de su funcionalidad, magnitud de capacidad, programas, entre otros elementos.

Asimismo, uno de los principales impactos lo ha ejercido el acceso al internet, para lo que se prevé su capacidad, cobertura, plataformas tecnológicas para su implementación dentro y fuera de las organizaciones.

Así, para la población objeto de la investigación se analiza la competitividad de las instituciones a partir de la presencia, condiciones, acceso, y uso en el proceso de las prácticas pedagógicas, la concepción y responsabilidad en el uso de las TIC como medio de comunicación y herramienta de apoyo a la formación mediante estrategias pedagógicas, didácticas, soportados en los alcances de la diversidad de programas básicos o de mayor complejidad que las instituciones adquieren y usan. 


\subsection{Factores de competitividad}

Martínez, Santero, Sánchez \& Marcos (2009) plantean que: La identificación de los principales factores de competitividad empresarial está ligada a la complejidad del propio concepto de competitividad. No obstante, hay un acierto sobre algunos factores determinantes en la mejora de la posición competitiva de la empresa, entre los que cabe destacar la innovación tecnológica, (...), la gestión de recursos humanos y el desarrollo de prácticas de gestión (p. 28).

Por otro lado, Garay (1998): "define que los factores empresariales se refieren a aquellos sobre los cuales la empresa tiene poder de decisión y que pueda controlar y modificar a través de su conducta" ( $p$. 569). Por consiguiente el uso de las TIC bien se establece como un medidor de las capacidades de las organizaciones, como un diferencial competitivo, que desde una mirada integral, su uso e implementación esta transversaliza aspectos de gestión, innovación e implicaciones del talento humano.

\subsubsection{Gestión}

Garay (1998) asegura que la gestión:

Incluye tareas administrativas típicas, toma de decisión, mínimo en el proceso de decisión y acción, finanzas, mercadeo, actividades pos venta, relaciones estrechas con los clientes, capacitación y planeación estratégica. Estas áreas forman parte del conjunto de factores críticos para el éxito en un mercado específico. (p 569). En este pilar recae la toma de decisiones de la alta gerencia.

\subsubsection{Innovación}

Competitividad desde la innovación permite afirmar que la innovación es un factor de productividad y en general de competitividad para ciertas empresas. La difusión de innovaciones, la "modernización" mediante la difusión de ciertas tecnologías son fenómenos que requieren ser separados a efectos de poder hacer su gestión y fomento. (Calva y Viniegra. 2007, p. 283)

\subsubsection{Talento humano}

Los recursos humanos se han convertido en uno de los principales factores de la competitividad empresarial en el marco de la economía del conocimiento. La gestión de los recursos humanos se define como el proceso para atraer, desarrollar y mantener una mano de obra conocimiento y la motivación necesarios para desarrollar las estrategias de las organizaciones y alcanzar sus objetivos. (Martínez et al., 2009, p. 32).

En este sentido, Mintzberg (1997), citado por Calderón y Castaño (2004), define la competitividad como la capacidad de una empresa para desarrollar ventajas comparativas que le permitan disfrutar y sostenerse en un sector específico, entendiéndose la ventaja como el valor que la empresa crea para sus clientes y el costo de la misma por crearlo. En este escenario, se puede considerar la competitividad empresarial desde una perspectiva interna donde se refiere a la competencia consigo misma a partir de la comparación de la eficiencia en el desarrollo de los diferentes procesos. La competitividad es el proceso encargado de generar fortalezas organizacionales para adaptarse y superar la variabilidad del entorno por medio de ventajas competitivas sustentables y sostenibles en el tiempo, con el fin de ser competitivas en el medio donde se desarrollan. Dicha actividad produce valor agregado y ventajas diferenciadoras entre las organizaciones que compiten en diferentes mercados. Por estas razones se debe considerar el concepto de competitividad como el conjunto de capacidades que tiene o desarrolla una organización para diferenciarse en un contexto determinado, mediante el aprovechamiento de ventajas comparativas, con el fin de competir eficientemente en diferentes contextos geográficos y en segmentos, dando respuesta al desafío competitivo afrontando amenazas y oportunidades del nuevo entorno.

Así mismo, Beltrán, Torres y otros (2006) interpretan el concepto de competitividad desde varias perspectivas. Inicialmente la definen como la competitividad de una nación que puede producir bajo condiciones de libre mercado, bienes y servicios que satisfagan el test de los mercados internacionales y simultáneamente incrementen los ingresos reales de los ciudadanos. De igual forma, la consideran como la capacidad de la empresa para formular e implantar estrategias que le permitan ampliar 0 conservar de forma duradera una posición en el mercado.

Según Reinel (2005), citado por Porter (2009), define la competitividad como la capacidad de la empresa para diseñar, desarrollar, producir y colocar sus productos en el mercado internacional en medio de la competencia con empresas de otros países, respondiendo ventajosamente en los mercados extranjeros, generando más valor agregado que sus competidores. Los elementos que constituyen este éxito son: la administración de flujos de producción, actividades de investigación y desarrollo, capacidad de incorporar cambios en la demanda, evolución de los mercados, capacidad de producir relaciones exitosas con otras empresas dentro de la cadena de valor. Por consiguiente, la competitividad es entendida como una característica intrínseca de un producto o de una firma que surge relacionada con el patrón de competencia vigente en cada mercado. De esta manera, constituye la capacidad de un país, empresa o sector para adaptarse en el tiempo en concordancia con las transformaciones que constantemente se producen en el medio.

\section{METODOLOGIA:}


El presente estudio es de naturaleza descriptiva con enfoque paradigmático cuantitativo, con estrategia no experimental, con diseño de investigación transeccional descriptivo, con método deductivo, y muestreo aleatorio simple (M.A.S).

Para la recolección de la información se tuvo en cuenta tres unidades de análisis que son: los rectores, docentes, y estudiantes del grado 10 y 11 de la jornada diurna de las Instituciones de educación pública de las comunas 7 y 8 del municipio de San José de Cúcuta. Allí se les aplicó la técnica encuesta, con diseño de preguntas de escala tipo Likert, y de selección múltiple con única respuesta a una muestra de 103 docentes, 313 estudiantes y 14 rectores, para este último se realizó un censo. A continuación se evidencia cálculo muestral realizado.

Fórmula (M.A.S) para población finita:

Para el caso de los docentes

Donde:

$\mathrm{N}=$ población $=140$ docentes

$Z=$ margen de confiabilidad $=95 \%$ que corresponde $a$ $1.96 \%$ desviación estándar.

$\mathrm{P}=$ probabilidad de que el evento ocurra $=0.5$

e: error de estimación $=0.05$

Para el caso de los estudiantes

$$
\begin{gathered}
n=\frac{1.96^{2} * 1685^{\star} 0.5^{*} 0.5}{(1685-1)^{\star} 0.05^{2}+1.96^{2}{ }^{*} 0.5^{\star} 0.5} \\
=313 \text { estudiantes }
\end{gathered}
$$

Donde:

$\mathrm{N}=$ Población $=2.2233$ estudiantes

$Z=$ Margen de confiabilidad $=95 \%$ que corresponde $a$ $1.96 \%$ desviación

estándar.

$\mathrm{P}=$ Probabilidad de que el evento ocurra $=0.5$

E: Error de estimación $=0.05$

\section{RESULTADOS Y DISCUSION:}

A partir de los instrumentos de recolección de la información, aplicados a 14 instituciones educativas públicas, con el fin de conocer la situación actual de la disponibilidad de las TIC, y cómo éstas estaban siendo empleadas en el proyecto pedagógico institucional, se pudo establecer que la educación media pública enfrenta grandes retos para
Tabla № 1.

\begin{tabular}{|c|c|c|c|c|c|}
\hline & & Frecuencia & Porcentaje & $\begin{array}{l}\text { Porcentaje } \\
\text { válido }\end{array}$ & $\begin{array}{l}\text { Porcentaje } \\
\text { acumulado }\end{array}$ \\
\hline \multirow[t]{5}{*}{ Válidos } & $\begin{array}{l}\text { Menos de } 10 \\
\text { computadoras }\end{array}$ & 6 & 42.9 & 42.9 & 42.9 \\
\hline & $\begin{array}{l}\text { Entre } 11 \text { a } 20 \\
\text { computadoras }\end{array}$ & 4 & 28.6 & 28.6 & 71.4 \\
\hline & $\begin{array}{l}\text { Entre } 21 \text { a } 30 \\
\text { computadoras }\end{array}$ & 2 & 14.3 & 14.3 & 85.7 \\
\hline & Ninguna & 2 & 14.3 & 14.3 & 100.0 \\
\hline & Total & 14 & 100.0 & 100.0 & \\
\hline
\end{tabular}

¿Cuantas computadoras dañadas posee la institución (incluso portátiles)?

Fuente: autores (2017)

El $(42.9 \%)$ de las I.E poseen menos de 10 computadoras dañadas, el (28.6\%) posee entre 11 a 20 computadoras, el (14.3\%) entre 21 a 30 computadoras.

En ese sentido se observa que las instituciones no poseen los equipos necesarios para el desarrollo de las prácticas pedagógicas, lo cual limita el aprendizaje de los estudiantes no despertando su interés en la búsqueda del conocimiento para la resolución de problemas.

Tabla No 2

¿Cómo es la presencia de las tecnologías en el proyecto pedagógico de la institución?

\begin{tabular}{llcccc}
\hline & & Frecuencia & Porcentaje & $\begin{array}{c}\text { Porcentaje } \\
\text { válido }\end{array}$ & $\begin{array}{c}\text { Porcentaje } \\
\text { acumulado }\end{array}$ \\
\hline Válidos & Muy buena & 4 & 3.9 & 3.9 & 3.9 \\
\hline & Buena & 46 & 44.7 & 45.1 & 49.0 \\
\hline & Regular & 39 & 37.9 & 38.1 & 87.3 \\
\hline & Mala & 11 & 10.7 & 10.8 & 98.0 \\
\hline & Muy mala & 2 & 1.9 & 2.0 & 100.0 \\
\hline Perdidos & Total & 102 & 99.0 & 100.0 & \\
\hline Total & Sistema & 1 & 1.0 & & \\
\hline
\end{tabular}

Fuente: autores (2017)

El $(44.7 \%)$ de los docentes consideraron que es buena la presencia de las tecnologías en el proyecto pedagógico de la institución, el (37.9\%) que es regular y el (10.7\%) la considera muy mala.

En la tabla 2, sumando las categorías regular y mala arroja como resultado que el $(48.6 \%)$, de las instituciones no existe una buena presencia de estas herramientas tecnológicas, en parte se debe a la falta de inversión del sector público en la educación, que provoca restricciones al acceso de la tecnología, en otros caso no existe una política institucional orientada al uso de las tecnologías en las practicas pedagógicas, lo cual provoca un estancamiento en los métodos de enseñanza, y por ende 
repercute en el bajo desarrollo de las habilidades cognitivas del estudiante.

\section{Tabla № 3.}

¿Considera que la formación y/o capacitación recibida en el uso de computadoras contribuyó a mejorar su práctica docente?

\begin{tabular}{|c|c|c|c|c|c|}
\hline & & Frecuencia & Porcentaje & $\begin{array}{l}\text { Porcentaje } \\
\text { válido }\end{array}$ & $\begin{array}{l}\text { Porcentaje } \\
\text { acumulado }\end{array}$ \\
\hline \multirow[t]{11}{*}{ Válidos } & Contribuyó totalmente & 36 & 35 & 35.0 & 35.0 \\
\hline & Contribuyó parcialmente & 43 & 41.7 & 41.7 & 76.7 \\
\hline & No contribuyó & 5 & 4.9 & 4.9 & 81.6 \\
\hline & $\begin{array}{l}\text { Recibí capacitaciòn, pero } \\
\text { como no utilizo, o utilizo }\end{array}$ & & & & \\
\hline & poco, computadoras e & 13 & 12.6 & 12.6 & 94.2 \\
\hline & internet en mi práctica & & & & \\
\hline & docente, no estoy en & & & & \\
\hline & condiciones de evaluar & & & & \\
\hline & No he recibido ninguna & 6 & 5.8 & 5.8 & 100.0 \\
\hline & capacitaciòn & & & & \\
\hline & Total & 103 & 100.0 & 100.0 & \\
\hline
\end{tabular}

Fuente: autores (2017)

El $(41.7 \%)$ de los docentes considera que la formación y/o capacitación recibida en el uso de computadoras contribuyó parcialmente a mejorar su práctica docente, el (35\%) afirma que contribuyó totalmente, el (12.62\%) recibieron capacitación, pero como no utilizan o hacen poco uso no están en condiciones de evaluar.

De la tabla 3 , se infiere que los docentes han realizado alguna preparación en cuanto a las TIC, que a pesar de haber sido cursos de corta duración en su mayoría contribuyó de manera significativa en el desarrollo de sus prácticas docentes, lo cual confirma que el uso y apropiación de estas herramientas brindan una mejor desenvolvimiento de las actividades, que ayudan a estimular la creatividad para el desarrollo de metodologías de enseñanza/aprendizajes que aporten significativamente al estudiante.

\section{Tabla No 4}

¿Cuál es el nivel de conocimientos que posee frente al término TIC (Tecnologías de la información y comunicación)?

\begin{tabular}{cccccc}
\hline & & Frecuencia & Porcentaje & $\begin{array}{c}\text { Porcentaje } \\
\text { válido }\end{array}$ & $\begin{array}{c}\text { Porcentaje } \\
\text { acumulado }\end{array}$ \\
\hline Válidos & Muy alto & 11 & 3.5 & 3.5 & 3.5 \\
\hline & Alto & 83 & 26.5 & 26.5 & 30.0 \\
\hline Regular & 182 & 58.1 & 58.1 & 88.2 \\
\hline Bajo & 29 & 9.3 & 9.3 & 97.4 \\
\hline Muy & 8 & 2.6 & 2.6 & 100.0 \\
bajo & & & & \\
\hline Total & 313 & 100.0 & 100.0 & \\
\hline
\end{tabular}

Fuente: autores (2017)
El nivel de conocimientos que poseen los estudiantes en cuanto a las TIC es regular con el (58.1\%), y el (9.3\%) posee un nivel bajo.

Sumando estas dos categorías denota que el (67.4\%) del estudiantado no poseen un buen nivel en cuanto al uso y apropiación de las TIC, algunas de las razones por las cuales se presenta este fenómeno es precisamente a la ausencia de herramientas tecnológicas en la institución, por otro lado, la variables ingresos, estrato y nivel educativo de los padres influyen en su educación, dado que al ser de estratos bajos su acceso a la tecnología es limitado.

\section{Tabla № 5.}

¿Cómo es la presencia de las tecnologías en el proyecto pedagógico de la institución?

\begin{tabular}{cccccc}
\hline & & Frecuencia & Porcentaje & $\begin{array}{c}\text { Porcentaje } \\
\text { válido }\end{array}$ & $\begin{array}{c}\text { Porcentaje } \\
\text { acumulado }\end{array}$ \\
\hline Válidos & Buena & 8 & 57.1 & 57.1 & 57.1 \\
\hline & Regular & 5 & 35.7 & 35.7 & 92.9 \\
\hline Mala & 1 & 7.1 & 7.1 & 100.0 \\
\hline Total & 14 & 100.0 & 100.0 & \\
\hline
\end{tabular}

Fuente: autores (2017)

El $(57.1 \%)$ de los rectores considera que es buena la presencia de las tecnologías en el proyecto pedagógico, el $(35.7 \%)$ que es regular y por último el $(7.1 \%)$ la considera mala.

La presencia de las tecnologías bajo la percepción de los rectores es considerada como buena, atribuyendo el hecho de que contar con las herramientas es sinónimo de apropiación en la práctica pedagógica, lo que refleja que las herramientas no son aprovechadas al máximo, sino como un simple apoyo a las actividades diarias.

\section{CONCLUSION:}

Analizado el impacto que ejerce el uso e implementación de las TIC en la competitividad de las organizaciones a partir de los actores y factores que involucra y para el caso tomado como referente en esta investigación referido a las instituciones de educación públicas, logra evidenciarse con los resultados obtenidos que estos centros no están preparados para llevar a cabo el proceso de integración de las TIC, dado que no cuentan con el apoyo necesario de las entidades competentes, por lo que las herramientas tecnológicas aparecen de forma parcial, y en general se emplean para reemplazar las actividades diarias, muy pocos docentes las emplean para promover espacio de aprendizajes, por ello, deberán reconocer la importancia que posee el componente tecnológico en el desarrollo de las habilidades cognitivas de los estudiantes, para que 
logren desenvolverse con éxito en el campo académico y laboral.

La gestión es uno de los factores más importante para cualquier área de una organización y más el sector educativo que posee la gran responsabilidad de ofrecer educación competitiva y de calidad, porque de ella depende que el estudiante alcance al final de su proceso de formación las competencias necesarias para insertarse en la educación superior o en la vida laboral. Para que los rectores puedan llevar a cabo dichas tareas, se hace necesario que cuenten con los recursos, en especial los económicos para el buen funcionamiento de las instalaciones, que va ligado a otras variables como la toma de decisiones, los programas de capacitación TIC docente, entre otros.

El sector educativo público enfrenta duros retos para alcanzar la competitividad, existen muchos factores que determinan la competitividad en el servicio educativo. En palabras de Santafé y Tuta (2016), en las instituciones educativas los factores de competitividad desde el punto de vista del desarrollo tecnológico y talento humano, permiten establecer debilidades y fortalezas con el fin de identificar los elementos diferenciadores frente a la competencia.

Los factores en que fueron evaluados las instituciones del presente estudio, corresponden a la gestión, innovación y talento humano, puesto que son claves para determinar que están haciendo las organizaciones desde adentro para ser competitivas, no pueden llegar a serlo cuando no poseen los recursos necesarios, debido a que se destinan pocos recursos por parte de las entidades territoriales.

La falta de ingresos en las instituciones ha creado la necesidad de buscar alternativas para generarlos, una de ellas es ampliar el cupo estudiantil, ocasionando sobre cupo en las aulas, obligando a que se tomen medidas drásticas como lo es el pico y placa, es decir los estudiantes deben turnarse para asistir a clases. La mayoría de los rectores son personas que poseen buena capacidad para tomar decisiones, pero no han tomado a aquellas que les ayude a mejorar el servicio educativo, como gestionar el desarrollo de las competencias digitales en sus docentes. No se puede innovar en la educación si las personas encargadas de llevar dicha innovación no saben cómo hacerlo a trabajarlo. Por consiguiente, Tuta y Santafé (2014), argumentan que la educación es el sistema central a través del cual la sociedad ha sustentado su crecimiento, la maduración del saber, apoyada en las diferentes disciplinas y herramientas específicamente las tecnológicas que enfocan el objetivo o meta establecida. En suma, en las 14 instituciones de educación investigadas no se evidencian los alcances y características de organizaciones competitivas mediante el uso de las TIC.

\section{REFERENCIAS:}

Beltrán, A. y Otros (2006). Mejorando la Competitividad de la Pyme. Desarrollo y Aplicación de Modelos de Gestión. 1a Edición. Universidad Externado de Colombia. Bogotá - Colombia.

Calderón, G. Y Castaño, G (2004). Investigación en Administración en América Latina. Universidad Nacional de Colombia. Sede Manizales. Bogotá Colombia.

Calva J..., Viniegra, G. (2007). Educación, ciencia, tecnología y competitividad: volumen 10 de agenda para el desarrollo. (p.p. 283). Universidad Autónoma de México, México D.F. Volumen 10. ISBN: 9703235425.

Escamilla, A., (2008). Las competencias básicas: claves y propuestas para su desarrollo en los centros. (pp. 79-197). Edición primera: Editorial GRAÒ, de IRIF, S.I. ISBN: 8478277714.

Garay L, (1998). Colombia: Estructura Industrial e internacionalización 1967-1996. (pp.569). Santafé de Bogotá, Colombia, Julio de 1998 (Tomo 1), ISBN: 958-8025-15-X

Guerrero, L. (2004). La Educación en el Contexto de la Globalización. (pp. 348). Boyacá, Colombia. Revista Historia de la Educación Latinoamericana. Universidad Pedagógica y Tecnológica de Colombia. ISSN: 0122-7238.

Instituto para el Desarrollo y la Innovación Educativa (IDIE) de la Organización de Estados Iberoamericanos para la Educación, la Ciencia y la Cultura (OEI). (2009). Integración de las TIC en la escuela: Indicadores de cualitativos y metodológicos de la investigación. Sao Paulo Brasil. (pp. 27,44, 72, 90, 106). ISBN: 978-84-7666-229-8 Depósito legal: M-41362-2011.

Martínez I, Santero R, Sánchez L \& Marcos M. (2009). Factores de competitividad de la pyme española 2008. (pp. 28,32). Fundación EOI Esc.Organiz.Industrial. ISBN: 978-84-88723-99-4.

Organización de los Estados Americanos (OEA). (2014). Sociedad del conocimiento. Washington, DC: OEA.

Porter, M. (2009). Ventaja Competitiva. Creación y Sostenimiento de un Desempeño Superior. Grupo Editorial Patria.6a reimpresión. México.

Santafé, A y Tuta, L. (2016). Competitividad. Estrategia emprendedora de posicionamiento en instituciones educativas. Editorial Redipe. Colombia ISBN. 978-958-59278-3-4.

Servicios TIC (2006). Universidad de Antioquia. Definición del concepto de TIC http://aprendeenlinea.udea.edu.co//ms/investigaci on $/ \mathrm{mod} /$ page/view.php?id=3118 consultada 18/03/2017) 
ISSN: 1794-9920 Enero - Abril de 2017

Volumen 17 Número 2, Año 2017 Págs. 110 - 119

Tuta, L y Santafé, A. (2014). Gerencia de la organización desde la óptica de la incertidumbre. Editorial Redipe. Colombia ISBN. 978-958-58073-9-6 